\title{
Baicalin Alleviates Oxidative Stress and Inflammation in Diabetic Nephropathy via Nrf2 and MAPK Signaling Pathway
}

Leyi Ma ${ }^{1}, *$

Fan $\mathrm{Wu}^{\mathrm{l}, *}$

Qingqing Shao'

Guang Chen ${ }^{2}$

Lijun $\mathrm{Xu}{ }^{\prime}$

Fuer Lu $\mathbb{D}^{\prime}$

'Institute of Integrated Traditional Chinese and Western Medicine, Tongji Hospital, Tongji Medical College, Huazhong University of Science and Technology, Wuhan, 430030, People's Republic of China; ${ }^{2}$ Department of Integrated Traditional Chinese and Western Medicine, Tongji Hospital, Tongji Medical College, Huazhong University of Science and Technology, Wuhan, 430030, People's Republic of China

*These authors contributed equally to this work
Correspondence: Fuer Lu Email felu@tjh.tjmu.edu.cn
Background: Oxidative stress and inflammation play essential roles in the development and progression of diabetic nephropathy (DN). Baicalin (BAI), a natural flavonoid, has been showed to have a renoprotective effect in various renal diseases. However, its underlying mechanisms in DN remain unclear. In this study, we explored the potential effects and underlying mechanisms of BAI on DN using a spontaneous DN model.

Methods: The protective effects of BAI on DN have been evaluated by detecting DNrelated biochemical indicators, kidney histopathology and cell apoptosis. After that, we examined the level of renal oxidative stress and inflammation to explain BAI's renoprotective effects. Then, Nrf2 pathway was tested to clarify its antioxidant activity, and kidney transcriptomics was conducted to elucidate its anti-inflammatory activity. Finally, Western blot was applied for final mechanism verification.

Results: Our results found that BAI effectively ameliorated diabetic conditions, proteinuria, renal histopathological changes and cell apoptosis in DN. BAI significantly improved the kidney levels of glutathione peroxidase (GSH-PX), superoxide dismutase (SOD) and catalase (CAT), and reduced malondialdehyde (MDA) level. Meanwhile, the infiltration of inflammatory cells including T-lymphocytes, T-helper cells, neutrophils and macrophages, and the mRNA levels of pro-inflammatory cytokines (IL-1 $\beta$, IL-6, MCP-1 and TNF $\alpha$ ) were also obviously inhibited by BAI. Afterward, Western blot found that BAI significantly activated $\mathrm{Nrf} 2$ signaling and increased the expression of downstream antioxidant enzymes (HO-1, NQO-1). Kidney transcriptomics revealed that the inhibition of MAPK signaling pathway may contribute to BAI's anti-inflammatory activity, which has also been verified in later experiment. BAI treatment did obviously inhibit the activation of canonical proinflammatory signaling pathway MAPK family, such as Erk1/2, JNK and P38.

Conclusion: In summary, our data demonstrated that BAI can treat DN by alleviating oxidative stress and inflammation, and its underlying mechanisms were associated with the activation of Nrf2-mediated antioxidant signaling pathway and the inhibition of MAPKmediated inflammatory signaling pathway.

Keywords: baicalin, diabetic nephropathy, oxidative stress, inflammation, Nrf2, MAPK

\section{Introduction}

Diabetic nephropathy (DN) is one of the major diabetic microvascular complications, which has surpassed glomerulonephritis as the primary cause of chronic kidney disease (CKD) in China. ${ }^{1,2}$ It was reported that more than $15-40 \%$ patients with diabetes mellitus (DM) may develop DN during their lifespan, ${ }^{3}$ and part of them eventually progresses to end-stage renal disease (ESRD). Currently, there is 
no satisfactory approach for the prevention and treatment of DN. Although tight glycemic control and blood pressure control have been proven to be effective, but many patients on oral hypoglycemic agents combined with angiotensin-converting enzyme inhibitors (ACEIs) or angiotensin receptor blockades (ARBs) continue to progress to ESRD, and patients' survival rate also has no significant improvement due to the limited efficacy and some side effects. ${ }^{2,4}$ Therefore, the development of novel agents is of great value in the treatment of DN.

The typical pathological characteristics of DN including tubular epithelial cell damage, glomerular sclerosis, apoptosis, inflammatory infiltration and renal interstitial fibrosis. ${ }^{5}$ To date, the pathogenesis of DN remains unclear. A number of evidences have suggested that oxidative stress and inflammation play the key roles in the development of $\mathrm{DN} .^{6-8}$ Mechanically, chronic hyperglycemia in DM patients is regarded as a trigger to initiate the downstream cascade. ${ }^{9}$ Briefly, hyperglycemia can induce the excessive production of reactive oxygen species (ROS), which invariably leads to oxidative stress. Then, oxidative stress arouses a series of metabolic and cellular disturbances, including lipid peroxidation, protein oxidation and DNA damage, ultimately lead to cell death. ${ }^{10}$ Besides, oxidative stress can also promote inflammatory response by stimulating the generation of related-growth factors, cytokines and transcription factors. ${ }^{11}$ Similarly, in diabetic patients, persistent hyperglycemia can also directly induce a chronic low-grade inflammatory status since diabetes has already been well recognized as a metabolic inflammation. ${ }^{12}$ Local chronic inflammatory stress not only directly causes organ damage and cell death but also impairs the antioxidant defense systems, thereby aggravating above-mentioned vicious cycle. $^{13,14}$ Taken together, there is a crosstalk between oxidative stress and inflammation in the progression of DN. Hyperglycemia-mediated oxidative stress and inflammation affect and promote each other, and in concert contribute to disease progression. ${ }^{15}$ In the treatment of DN, anti-oxidative stress or anti-inflammation therapies have been widely investigated and proven to have certain effects. ${ }^{16,17}$ Given that, compared with single anti-oxidative stress or antiinflammation therapies, multiple pathways (anti-oxidative and anti-inflammation)-targeted strategy may exert a better efficacy and have gained substantial interest.

Baicalin (BAI), one of the major bioactive flavonoids from the root of medicinal plant Scutellaria baicalensis Georgi (S. baicalensis), which has been proven to possess various pharmacological effects, such as antidiabetic, antioxidant, anti-inflammatory and anti-cancer properties. ${ }^{18-20}$ Studies have demonstrated that BAI can protect against DN by inhibiting progressive renal fibrosis, and the related mechanisms were associated with the inhibition of NF- $\mathrm{KB}$ signaling. ${ }^{21-23}$ Since organ fibrosis is a result of chronic stress and always occurs in the middle and late stages of chronic diseases, fibrogenesis may not be the foremost promoter in the early stage of DN. ${ }^{24}$ However, current studies about BAI on DN were mainly focused on the renal fibrosis, few researches were conducted to explore the effects of BAI on the upstream events in DN: oxidative stress and inflammation. Intriguingly, a recent clinical trial studied the effects of baicalin on DN patients, and it was found that BAI improves the renal function and delays the disease progression in DN patients via anti-inflammatory and antioxidant pathways. ${ }^{25}$ However, further in-depth investigation into model animals is still lacking. Based on oxidative stress and inflammation, clarifying the underlying mechanisms of BAI's protective effects on DN is significant to the development of novel agents.

Given that, we hypothesized that BAI could prevent DN by alleviating oxidative stress and inflammation. In order to verify our hypothesis, we performed in vivo experiments to evaluate the effects of BAI on DN. After that, oxidative stress and inflammation levels were determined to explain the BAI's renoprotective property. Finally, several crucial signaling pathways were verified to elucidate the underlying molecular mechanisms. Our results shed light on BAI as a potential natural product that targets oxidative stress and inflammation to treat DN.

\section{Materials and Methods}

\section{Reagents and Chemicals}

Baicalin was obtained from Shanghai Yuanye BioTechnology Co., Ltd. (Shanghai, China), and it was resuspended using distilled water to $40 \mathrm{mg} / \mathrm{mL}$ for the animal experiments. Antibodies against P38, Bax, Bcl-2, Caspase-3, Erk1/2, Nrf2, HO-1 and $\beta$-action were purchased from Proteintech (Wuhan, China). JNK, p-JNK and all secondary antibodies used in Western blot were obtained from Cell Signaling Technology (Beverly, MA, USA). p-P38, p-Erk1/ 2, NQO-1, CD3 and MPO antibodies were from ABclonal Technology (Wuhan, China). CD68 antibody was provided by Abcam (Cambridge, MA). CD4 antibody was obtained from Santa Cruz Biotechnology (Santa Cruz, CA). Trizol, Hifair $^{\mathrm{TM}}$ II 1st Strand cDNA Synthesis Super Mix and Hieff $^{\circledR}$ qPCR SYBR ${ }^{\circledR}$ Green Master Mix were from Yeasen 
(Shanghai, China). The TUNEL apoptosis and immunohistochemistry kits were obtained from Wuhan Gugeshengwu Technology Co., Ltd. (Wuhan, China). All other regular reagents were obtained from Wuhan Gugeshengwu Technology Co., Ltd. unless otherwise specified.

\section{Animal Experiment}

The animal experiment was overseen and approved by the Animal Ethics Committee of Tongji Hospital of Tongji Medical College of Huazhong University of Science and Technology (HUST) according to the Guideline for Ethical Review of Animal Welfare (GB/T 35892-2018). Sevenweek-old male $\mathrm{db} / \mathrm{db}$ and $\mathrm{db} / \mathrm{m}$ mice were provided by Nanjing Biomedical Research Institute of Nanjing University and housed in the experimental animal center (SPF-grade) under conditions of $12 \mathrm{~h}$ dark/light cycle, $60 \pm$ $5 \%$ relative humidity and $20 \pm 2{ }^{\circ} \mathrm{C}$ environmental temperature. After one-week acclimation, $\mathrm{db} / \mathrm{db}$ mice were randomly divided into two groups: model group $(n=8)$ and BAI group (400 mg/kg, $\mathrm{n}=8$ ), while $\mathrm{db} / \mathrm{m}$ mice were assigned as control group $(n=8)$. In control and model groups, the mice were gavaged daily $(10 \mathrm{~mL} / \mathrm{kg}$ body weight) with distilled water, and in BAI group, the mice were administered daily with BAI suspension ( $40 \mathrm{mg} / \mathrm{mL}$ ) in the same dose. Due to natural flavonoids have common low oral-bioavailability characteristics, the dose was chosen based on previous studies to ensure its efficacy. ${ }^{26,27}$ The drug treatment lasted for the next 8 weeks until the end of this study. The experimental protocol is illustrated in Figure 1A. During the study, body weight was measured every three days and fasting blood glucose (FBG) was measured weekly. At the end of the experiment, all mice were anaesthetized with $1 \%$ pentobarbital (65 $\mu \mathrm{L} / 10$ g, i.p.). After collecting blood sample, the mice were euthanized with $\mathrm{CO}_{2}$.

\section{Measurement of Diabetic Parameters}

During the last week of the study, glucose and insulin tolerance tests were conducted. Briefly, all mice were fasted overnight and intraperitoneally injected with glucose (i.p., $0.75 \mathrm{~g} / \mathrm{kg}$ ) for glucose tolerance test (GTT). For insulin tolerance test (ITT), the mice were fasted $6 \mathrm{~h}$ and intraperitoneally injected with insulin (i.p., $1.0 \mathrm{U} / \mathrm{kg}$ ). The tail vein blood glucose was measured at $0,15,30,60$ and $120 \mathrm{~min}$ after glucose or insulin administration by glucose strip (ACCU-CHEK Performa, Roche). After collecting blood sample, serum insulin levels were determined by ELISA kit (Bioswamp Life Science Lab, Wuhan, China) followed manufacturer's protocol. Homeostasis model assessment insulin resistance (HOMAIR) index was calculated according to the following formula:

$\mathrm{HOMA}-\mathrm{IR}=\mathrm{FBG}(\mathrm{mM}) \times$ fasting $\operatorname{insulin}(\mathrm{mU} / \mathrm{L}) / 22.5$

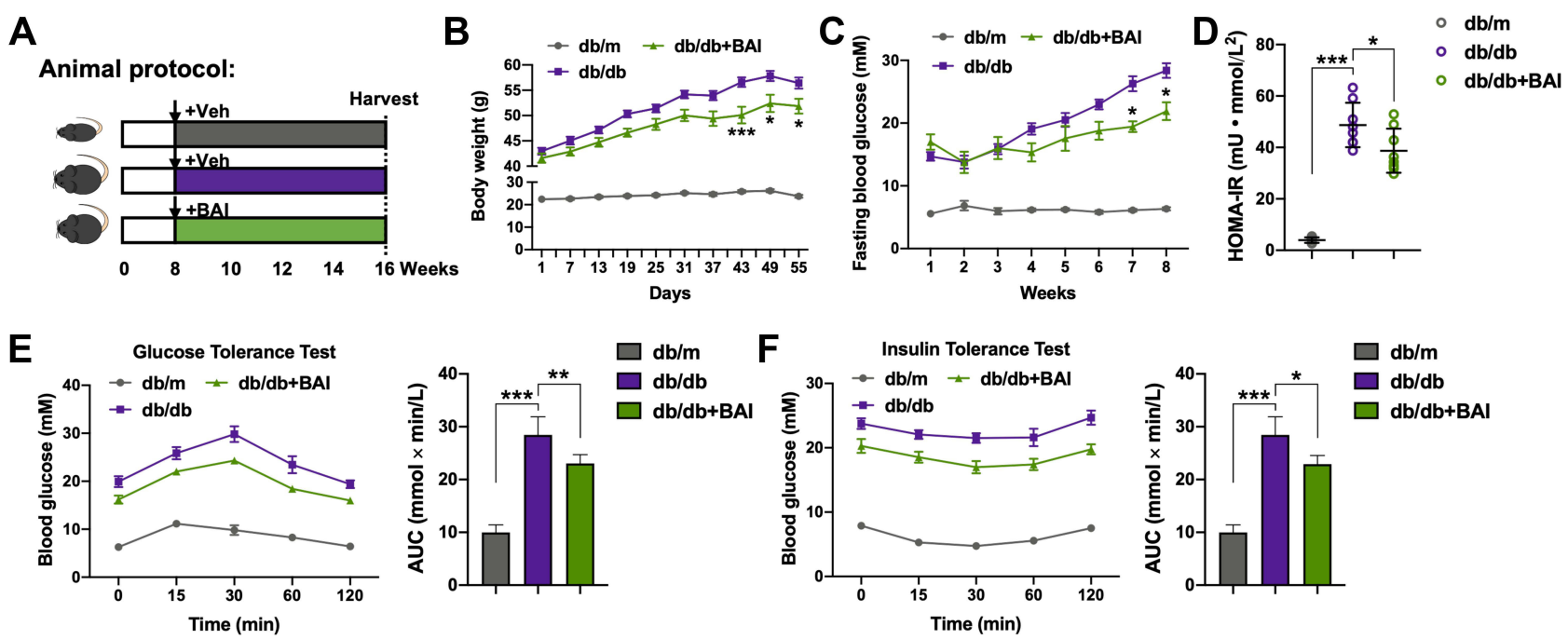

Figure I BAl administration significantly ameliorates diabetic conditions in db/db mice. (A) Animal experimental protocol of this study. (B) Body weight of mice was recorded every 3 days during the experiment. $(n=8)(\mathbf{C})$ Fasting blood glucose of mice was recorded weekly during the experiment. ( $n=8)(\mathbf{D})$ HOMA-IR index was calculated according to standard formula: HOMA-IR= FBG $(\mathrm{mM}) \times$ fasting insulin $(\mathrm{mU} / \mathrm{L}) / 22.5$. $(\mathrm{n}=8)(\mathrm{E})$ For GTT, mice were fasted overnight and intraperitoneally injected with glucose (i.p., $0.75 \mathrm{~g} / \mathrm{kg}$ ), blood glucose was measured at 0, 15, 30, 60 and 120 min after glucose administration; the bar graph represents average area under the curve. $(\mathrm{n}=6)(\mathbf{F})$ For ITT, mice were fasted overnight and intraperitoneally injected with insulin (i.p., I.0 U/ $/ \mathrm{kg}$ ), blood glucose was measured at 0 , I5, 30 , 60 and I 20 min after insulin administration; the bar graph represents average area under the curve. $(\mathrm{n}=6)$ All data are presented as means $\pm S D$. $*_{p}<0.05, * * p<0.01, * * * p<0.001$. 


\section{Kidney Function Tests}

In the last week of drug treatment, mice urine samples of $24 \mathrm{~h}$ were collected individually using metabolic cages. After that, urine albumin levels were measured by ELISA kit (Bioswamp Life Science Lab, Wuhan, China). Urine and serum creatinine and serum urea nitrogen (BUN) were determined by Creatinine (Cr) and Urea assay kits (Nanjing Jiancheng Bioengineering Institute, Nanjing, China) according to the corresponding manufacturer's instructions. Urine albumin excretion rate (AER) was calculated according to the following formula: AER $(\mu \mathrm{g} / 24 \mathrm{~h})=$ Urine albumin $(\mu \mathrm{g} / \mathrm{mL}) \times 24$ $\mathrm{h}$ urine volume $(\mathrm{mL} / 24 \mathrm{~h})$. Urine albumin/creatinine ratio (ACR) was calculated according to the following formula:

$$
\begin{aligned}
\operatorname{ACR}(\mathrm{mg} / \mathrm{mmol}) & =\text { Urine albumin }(\mu \mathrm{g} / \mathrm{mL}) \\
& \times 1000 / \text { Urine creatinine }(\mu \mathrm{M})
\end{aligned} .
$$

Creatinine clearance rate $(\mathrm{Ccr})$ was calculated according to the following formula:

$$
\begin{aligned}
\operatorname{Ccr}(\mathrm{mL} / \mathrm{min} / \mathrm{kg}) & =\left[\begin{array}{l}
\text { Urine creatinine }(\mu \mathrm{M}) \\
\times 24 \mathrm{~h} \text { rine volume }(\mathrm{mL}) \times 1000
\end{array}\right] \\
/ & {\left[\begin{array}{l}
\text { Serum creatinine }(\mu \mathrm{M}) \\
\times \text { body weight }(\mathrm{g}) \times 1440(\mathrm{~min})
\end{array}\right] }
\end{aligned}
$$

\section{Kidney Histology and Electron Microscopy}

Paraffin-embedded kidney slides were stained with periodic acid Schiff (PAS) according to the standard protocol for histological analysis. Firstly, kidney images were taken by an Olympus BX51 system (Olympus, Japan). Then, images were scanned and profile areas were traced using Image J. The glomerular area was calculated based on the average area of 10 glomeruli in each group, and the mesangial fraction was defined as a ratio between PASpositive and nuclei-free area to PAS-positive area (including nuclei area).

To uncover the ultrastructure changes in the glomerulus, transmission and scanning electron microscopy (Hitachi, Tokyo, Japan) were also applied for observing ultrastructure histopathology.

\section{Determination of Oxidative Stress Indicators}

Frozen kidney samples were homogenized in PBS, and the supernatant of tissue homogenates was employed to estimate oxidative stress indicators. Glutathione peroxidase
(GSH-PX), superoxide dismutase (SOD), catalase (CAT) and malondialdehyde (MDA) assay kits were obtained from Nanjing Jiancheng Bioengineering Institute (Nanjing, China). These biochemical indicators were examined according to the instructions.

\section{Immunohistochemistry Staining}

Paraffin-embedded slides were dewaxed by dimethylbenzene, polarized with descending concentrations of alcohol, and rinsed with deionized water. Endogenous peroxidase activity was blocked by incubating slides in $3 \% \mathrm{H}_{2} \mathrm{O}_{2}$ at room temperature for 30 minutes after antigen retrieval. The $20 \%$ normal goat serum was used to block the slides for $1 \mathrm{~h}$. Primary antibodies were applied overnight at $4{ }^{\circ} \mathrm{C}$ followed by incubation of HRP-conjugated secondary antibody for $60 \mathrm{~min}$ at room temperature. The slides were visualized by $\mathrm{DAB}$ and counterstained with hematoxylin. Images were taken by an Olympus BX51 system and analyzed by Image $\mathrm{J}$ software (National Institutes of Health, USA).

\section{TUNEL Staining}

According to the manufacturer's protocol, kidney slides were used for TUNEL apoptosis kit (Gugeshengwu Technology, Wuhan, China). An Olympus BX51 system (Olympus, Japan) was used to observe TUNEL-positive cells.

\section{Transcriptomics Analysis}

The transcriptomics analysis was conducted by Seqhealth Technology Co., LTD (Wuhan, China). Total RNA of fresh kidney tissue was isolated using Trizol reagent. The RNA concentration and purity were determined by Nanodrop ${ }^{\mathrm{TM}}$ OneC spectrophotometer (Thermo Fisher Scientific Inc., US). RNA integrity was tested by $1.0 \%$ agarose gel electrophoresis. Two microgram total RNAs were used for stranded RNA sequencing library preparation using KC-Digital ${ }^{\mathrm{TM}}$ Stranded mRNA Library Prep Kit for Illumina ${ }^{\circledR}$ (Catalog NO. DR08502, Wuhan Seqhealth Co., Ltd. China) following the manufacturer's instruction. After cDNA library construction, the concentration and quality of libraries were evaluated by Qubit 2.0 (Life Technologies, US) and agarose gel electrophoresis. Afterward, 200-500 bp products were enriched, quantified, and finally sequenced in HiSeq X10 system (Illumina, San Diego, CA, USA).

For the RNA-seq data analysis, raw data were first filtered by Trimmomatic software (version: 0.36) in which low-quality reads were discarded, and adaptor 
sequences were trimmed. Clean reads from each sample were mapped to the GRCm38 mouse reference genome using the Star program (2.3.0). A corrected P-value cutoff of 0.05 and fold change cutoff of 1 were used to judge the statistical significance of the differentially expressed genes (DEGs). Gene ontology (GO) and Kyoto Encyclopedia of Genes and Genomes (KEGG) enrichment analysis of DEGs was conducted using Metascape database.

\section{Real-Time Quantitative Polymerase Chain Reaction PCR (RT-qPCR)}

Tissue RNA was extracted using Trizol reagent according to the standard protocol. After reverse-transcription, RT-qPCR was performed on LightCycler ${ }^{\circledR} 96$ system (Roche Diagnostics, Mannheim, Germany). The mRNA level of target genes was normalized and analyzed by the $2^{-\Delta \Delta \mathrm{CT}}$ method. Sequences of the primers used in this study are listed in Table 1.

\section{Western Blot Analysis}

Total proteins were extracted by RIPA buffer supplemented with $1 \%$ of PMSF and protease inhibitor cocktail followed the standard protocol, and then protein concentrations were quantified using a bicinchoninic acid (BCA) protein assay kit. Equal amounts of protein extracts were loaded on SDSPAGE $(80 \mathrm{~V}, 0.5 \mathrm{~h}$ and then $120 \mathrm{~V}, 1 \mathrm{~h})$ and electrotransferred to a $0.45 \mu \mathrm{m}$ nitrocellulose membrane or $0.22 \mu \mathrm{m}$ PVDF membrane $(280 \mathrm{~mA}, 1 \mathrm{kDa} / \mathrm{min})$. The membranes were blocked with $5 \%$ nonfat milk for $1 \mathrm{~h}$ at room temperature and incubated with primary antibodies overnight at $4{ }^{\circ} \mathrm{C}$. On the next day, fluorescence-conjugated secondary antibodies were applied to the membranes for $1 \mathrm{~h}$ at room temperature. The membranes were visualized with Odyssey Infrared Imaging (LI-COR Biosciences, USA). Target proteins were normalized to $\beta$-actin and quantified by Image $J$ software.

\section{Statistical Analysis}

Statistical analyses were performed following this rule: Firstly, the normality of data is tested by QQ plot together with
Shapiro-Wilk test; Secondly, ANOVA test with Tukey posthoc analysis was used for data following the normal distribution and Kruskal-Wallis test with Dunn's post hoc test for skewed data (not obeying the normal distribution). Data fit the normal distribution are presented as means $\pm \mathrm{SD}$, and skewed data are presented as median (min-max). Statistics were analyzed using the GraphPad Prism 8.0 and SPSS 24.0, and $p<$ 0.05 was considered as statistically significant.

\section{Results}

\section{BAI Administration Significantly Ameliorates Diabetic Conditions in Db/ Db Mice}

In this work, $\mathrm{db} / \mathrm{db}$ mice were used as the spontaneous $\mathrm{DN}$ model. Firstly, we examined the diabetic indicators under BAI treatment. As shown in Figure 1B, BAI administration obviously prevented weight gain compared to model group at the end of the experiment (Control group vs model group $p<0.001$, BAI group vs model group $p=0.022$ ). Compared with control group, fasting blood glucose (FBG) of model group was remarkedly elevated, while BAI administration significantly reduced FBG (Control group vs model group $p<0.001$, BAI group vs model group $p=0.013$ ) (Figure 1C). After collecting samples, the fasting insulin level was also detected. It was found that model and BAI groups all showed a reactive increased insulin level compared to control group, but there was no difference in fasting insulin level between model and BAI groups (Control group vs model group $p<0.001$, BAI group vs model group $p=0.7637$ ) (Supplementary Figure 1). This result indicated that BAI may have no effects on insulin secretion. Due to the improved FBG conditions, the homeostasis model assessment insulin resistance (HOMA-IR) index was still decreased by BAI administration compared to model group (Control group vs model group $p<0.001$, BAI group vs model group $p=0.0256$ ) (Figure 1D). In addition, the glucose metabolism state was also assessed by glucose and insulin tolerance tests. Consistent with the above results, BAI administration can increase

Table I Primers Used for RT-qPCR

\begin{tabular}{|c|c|c|}
\hline Gene & Forward (5'-3') & Reverse (5'-3') \\
\hline$I L-I \beta$ & TCATTGTGGCTGTGGAGAAG & AGGCCACAGGTATTTTGTCG \\
\hline IL-6 & ССССААТTТССААТGСТСТСС & CGCACTAGGTTTGCCGAGTA \\
\hline$M C P-I$ & CGCACTAGGTTTGCCGAGTA & TGTCTGGACCCATTCCTTCTTG \\
\hline$T N F \alpha$ & СССТСАСАСТСАСАААССАСС & CTTTGAGATCCATGCCGTTG \\
\hline$\beta$-actin & GTGACGTTGACATCCGTAAAGA & GAAACAGTCCGCCTAGAAGCAC \\
\hline
\end{tabular}


glucose clearance rate and improve insulin sensitivity (GTT: Control group vs model group $p<0.001$, BAI group vs model group $p=0.0034$; ITT: Control group vs model group $p<$ 0.001 , BAI group vs model group $p=0.0114$ ) (Figure $1 \mathrm{E}$ and F). Although BAI-treated $\mathrm{db} / \mathrm{db}$ mice are still far from normal mice in terms of diabetes status, but compared BAI group with model mice, it was apparent that BAI administration significantly ameliorates diabetic conditions in $\mathrm{db} / \mathrm{db}$ mice. Therefore, there is no doubt that BAI possesses antidiabetic effect. Taken together, all these data demonstrated that BAI administration significantly ameliorates diabetic conditions in $\mathrm{db} / \mathrm{db}$ mice.

\section{BAl Administration Reduces Proteinuria and Improves Histopathological Changes in $\mathrm{DN}$}

Under normal conditions, $\mathrm{db} / \mathrm{db}$ mice will spontaneously develop diabetic kidney injury over time. Based on that, we further investigated the protective effects of BAI on kidney function through examining various biochemical indicators. Compared with control group, mice from model group displayed typical symptom of microalbuminuria (Figure 2A), which also suggested that our DN model is successful. BAI treatment significantly decreased urine albumin level, urine albumin/creatinine ratio (ACR) and urine albumin excretion rate (AER) compared to model group (Urinary albumin: Control group vs model group $p<0.001$, BAI group vs model group $p<0.001$; ACR: Control group vs model group $p<0.001$, BAI group vs model group $p=0.0021$; AER: Control group vs model group $p<0.001$, BAI group vs model group $p<0.001$ ) (Figure 2A). Compared with control group, $24 \mathrm{~h}$ urine volume in model and BAI group were also obviously increased, in which BAI administration led to a trend of reduction in urine volume although it was not statistically significant (Control group vs model group $p<0.001$, BAI group vs model group $p=0.3624$ ) (Supplementary Figure 2). Additionally, we found that there was no difference in serum urea nitrogen (BUN), serum creatinine and creatinine clearance rate (Ccr) between different groups
A

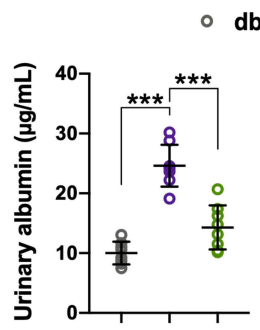

- $\mathrm{db} / \mathrm{m}$

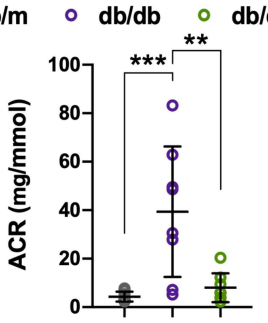

db/db+BAl

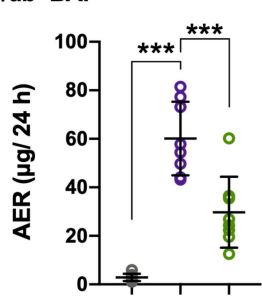

B

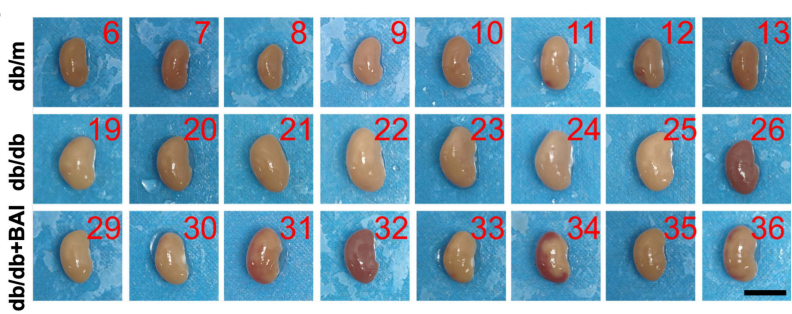

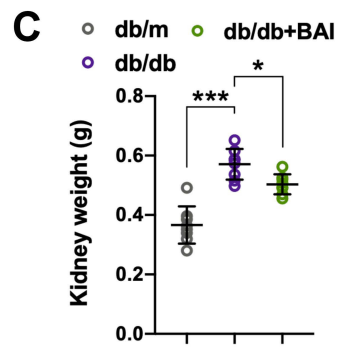
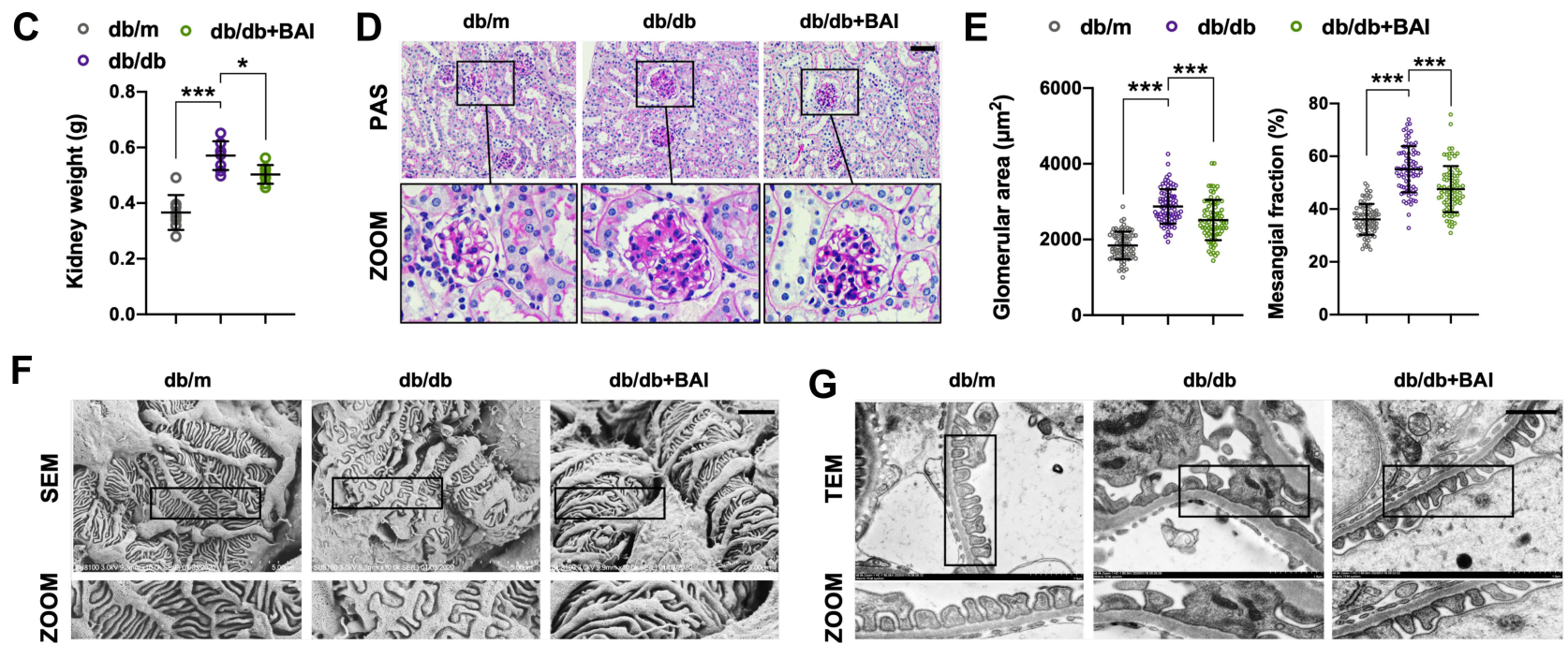

Figure 2 BAl administration reduces proteinuria and improves histopathological changes in DN. (A) The urine albumin level, urine albumin/creatinine ratio (ACR) and urine albumin excretion rate (AER) of different groups. $(n=8)(B)$ Kidney images of different groups. Scale bar, I $\mathrm{cm}(n=8)(\mathbf{C})$ Kidney weight of different groups. ( $n=8)(\mathbf{D})$ Representative kidney PAS staining of different groups. Scale bar, $50 \mu \mathrm{m}$. $(n=8)(\mathbf{E})$ Glomerular area and mesangial fraction of different groups. ( $\mathrm{n}=8)(\mathbf{F})$ Representative kidney SEM images of different groups. Scale bar, $2 \mu \mathrm{m}$. $(\mathrm{n}=8)(\mathbf{G})$ Representative kidney TEM images of different groups. Scale bar, I $\mu \mathrm{m}$. ( $\mathrm{n}=8)$ All data are presented as means \pm SD. $* p<0.05, * * p<0.01$, $* * * p<0.001$. 
(BUN: Control group vs model group $p=0.1334$, BAI group vs model group $p=0.5229$; Scr: Control group vs model group $p=0.1289$, BAI group vs model group $p=$ 0.7232; Ccr: Control group vs model group $p=0.7153$, BAI group vs model group $p=0.9914$ ) (Supplementary Figure 2). This phenomenon was in our expectation. In this study, the reason why we employed a relatively short intervention time was to make DM mice with typical proteinuria but not kidney dysfunction (early stage of DN). These results indicated that BAI can exert an obvious effect in reducing proteinuria.

Morphologically, the kidney size and weight of model group was apparently larger than that of control group, and BAI treatment decreased kidney size and weight in model mice (Control group vs model group $p<0.001$, BAI group vs model group $p=0.0375$ ) (Figure $2 \mathrm{~B}$ and $\mathrm{C}$ ). However, it was found that kidney indexes (kidney weight/body weight ratio) of model and BAI groups were all smaller than that of control group, and there was no difference between these two groups (Control group vs model group $p<0.001$, BAI group vs model group $p=0.8462$ ) (Supplementary Figure 3). This result was also related to our early DN model. In addition to persistent proteinuria, histopathological changes like glomerular hypertrophy, mesangial matrix expansion, glomerular basement membrane (GBM) thickening and podocyte injury are also the important features in DN. Histological analysis of PAS staining showed that BAI significantly attenuated glomerular hypertrophy and mesangial matrix expansion compared to model group (Glomerular area: Control group vs model group $p<0.001$, BAI group vs model group $\mathrm{p}<$ 0.001; Mesangial fraction: Control group vs model group $p<0.001$, BAI group vs model group $p<0.001$ ) (Figure 2D and E). Scanning and transmission electron microscopy (SEM and TEM) images showed significant histological changes, such as podocyte detachment, foot process effacement and GBM thickening in DN mice, which were obviously reversed by BAI treatment (Figure $2 \mathrm{~F}$ and $\mathrm{G}$ ). Collectively, our data suggested that BAI can reduce proteinuria and improve histopathological changes in $\mathrm{DN}$ mice.

\section{BAI Administration Suppresses Cell Apoptosis in DN}

It has been proved that cell apoptosis plays a key role in the development and progression of DN. ${ }^{28}$ In DN, apoptosis can be triggered by various factors, including hyperglycemia, oxidative stress and inflammation, and it can occur in multiple cell types, like tubular epithelial cells, endothelial and interstitial cells. ${ }^{29}$ In order to determine whether BAI could affect cell apoptosis in DN, terminal deoxynucleotidyl transferase dUTP nick end labeling (TUNEL) staining was used to observe apoptotic cells. TUNEL staining results revealed that apoptosis level of model group was significantly induced compared to that of control group (green area), and BAI administration suppressed excessive cell apoptosis compared to model group (Figure 3A). Meanwhile, we examined the expression levels of apoptosis-related proteins, including Bax, Bcl-2 and cleaved caspase-3. Consistently, model group exhibited increased expression of pro-apoptotic proteins, Bax and cleaved caspase-3, and reduced expression of antiapoptotic protein, Bcl-2, which was reversed by BAI administration (Cleaved caspase-3: Control group vs model group $p<0.001$, BAI group vs model group $p<0.001$; Bax: Control group vs model group $p<0.001$, BAI group vs model group $p<0.001$; $\mathrm{Bcl}-2$ : Control group vs model group $p<0.001$, BAI group vs model group $p<0.001$ ) (Figure 3B and C). Together, these data suggested that BAI administration can suppress cell apoptosis in DN.

\section{Baicalin Administration Alleviates Oxidative Stress in DN}

To evaluate the effects of BAI on oxidative stress, we tested the kidney levels of oxidative stress indicators, including glutathione peroxidase (GSH-PX), superoxide dismutase (SOD), catalase (CAT) and malondialdehyde (MDA). As shown in Figure 4, compared with model group, the levels of GSH-PX, SOD and CAT were significantly improved, and the level of MDA was significantly reduced in BAI group (SOD: Control group vs model group $p=0.006$, BAI group vs model group $p=0.0438$; GSH-PX: Control group vs model group $p=0.0071$, BAI group vs model group $p=$ 0.0259; CAT: Control group vs model group $p<0.001, \mathrm{BAI}$ group vs model group $p=0.0197$; MDA: Control group vs model group $p=0.0034$, BAI group vs model group $p=$ 0.032). These results demonstrated that BAI administration can alleviate oxidative stress injury via enhancing antioxidant enzyme activities.

\section{Baicalin Administration Alleviates Inflammatory Response in DN}

Next, we investigated the effects of BAI on local inflammatory response in DN. First, inflammatory cell infiltration was detected using immunohistochemistry staining. 


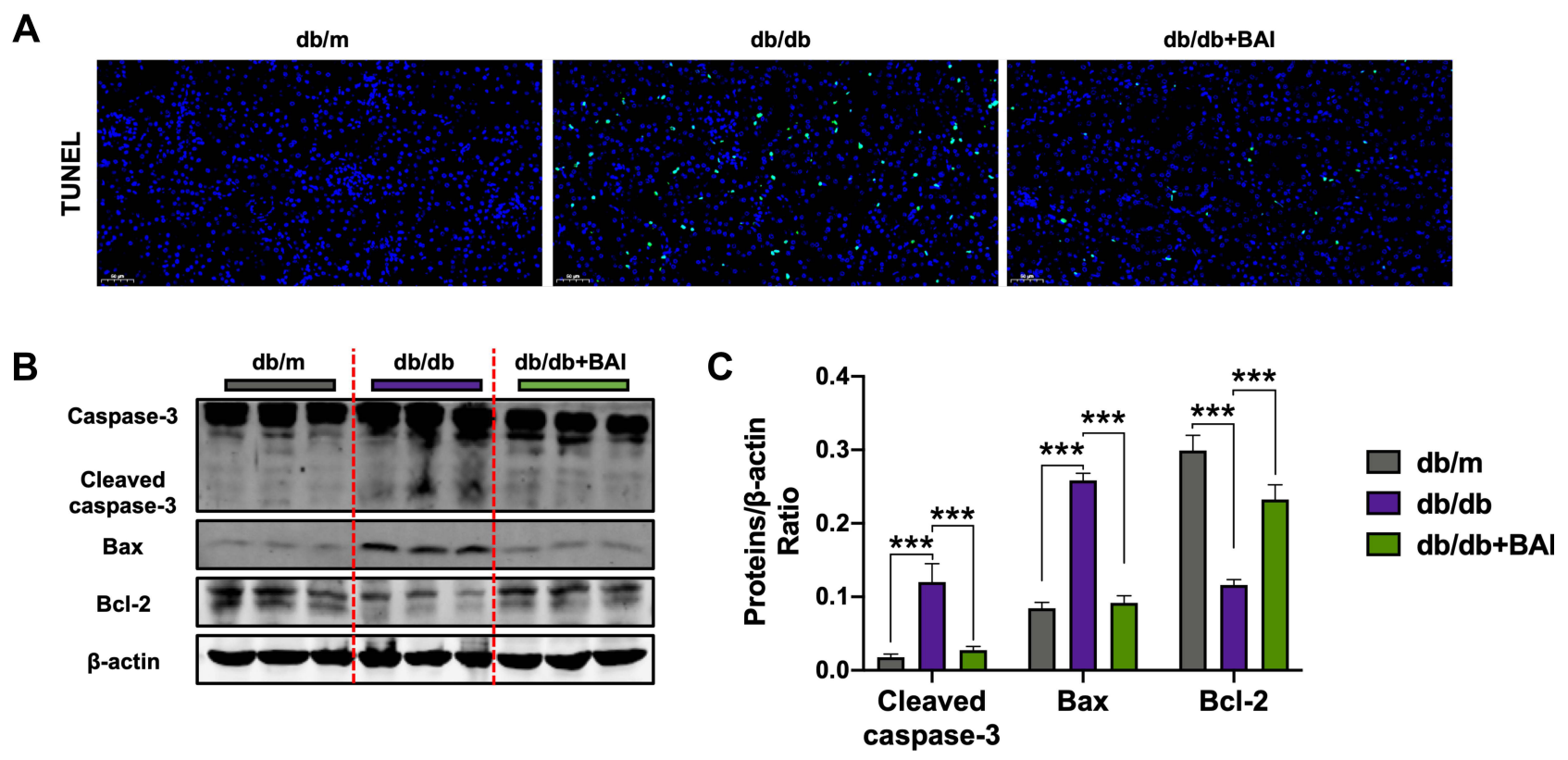

Figure 3 BAl administration suppresses cell apoptosis in DN. (A) Representative kidney TUNEL staining of different groups. Scale bar, $50 \mu$ m. ( $n=6$ ) (B) Representative Western blots for caspase-3, cleaved caspase-3, Bax and Bcl-2 protein expressions in kidney tissue extracts. ( $\mathrm{n}=6)(\mathbf{C})$ The quantification of cleaved caspase-3, Bax and Bcl-2 protein Western blots. All data are presented as means \pm SD. $* * * p<0.001$.
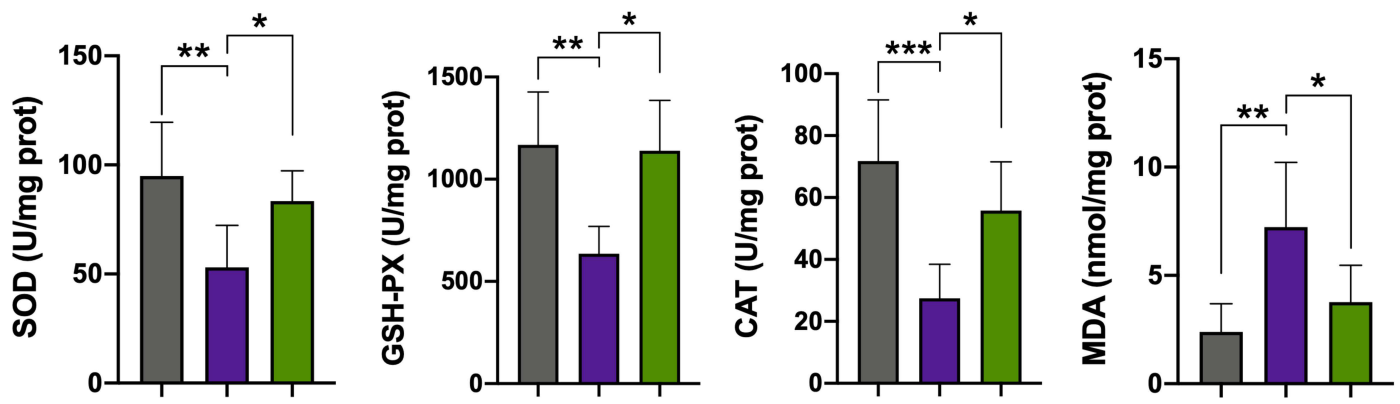

$\mathrm{db} / \mathrm{m}$

$\mathrm{db} / \mathrm{db}$

$\mathbf{d b} / \mathbf{d b}+\mathbf{B A l}$

Figure 4 Baicalin administration alleviates oxidative stress in DN. The levels of SOD, GSH-PX, CAT and MDA in kidney tissue extracts of different groups. ( $\mathrm{n}=6$ ) All data are presented as means $\pm S D$. * $p<0.05$, **p $<0.01$, ***p $<0.001$.

According to Figure 5A, it was apparent that more inflammatory cells, including T-lymphocytes (CD3-positive), $\mathrm{T}$ helper cells (CD4-positive), neutrophils (MPOpositive) and macrophages (CD68-positive), were observed in model group, whereas few inflammatory cells were detected in control group. Compared with model group, BAI group showed a significant reduction in various inflammatory cell infiltration. Then, the mRNA levels of typical pro-inflammatory cytokines, such as $I L$ $1 \beta, I L-6, M C P-1$ and $T N F \alpha$, were also determined by RTqPCR. Similarly, in DN mice, the mRNA levels of $I L-1 \beta$, $I L-6, M C P-1$ and $T N F \alpha$ were obviously elevated compared to control group, and BAI treatment decreased their transcriptional levels ( $I L-1 \beta$ : Control group vs model group $p=0.0017$, BAI group vs model group $p=0.0232 ; I L-6$ :
Control group vs model group $p<0.001$, BAI group vs model group $p<0.001 ; M C P-1$ : Control group vs model group $p=0.0013$, BAI group vs model group $p=0.0466$; $T N F \alpha$ : Control group vs model group $p=0.0056, \mathrm{BAI}$ group vs model group $p=0.0307$ ) (Figure 5B). Therefore, these findings showed that baicalin administration can exert an ameliorative effect on renal inflammatory response in $\mathrm{DN}$.

\section{Baicalin Administration Activates Nrf2-Mediated Antioxidant Pathway in DN}

After confirming the antioxidant effect of BAI, we further explored the potential mechanisms. Nuclear factor erythroid-derived 2-related factor 2 (Nrf2) plays a pivotal 
A

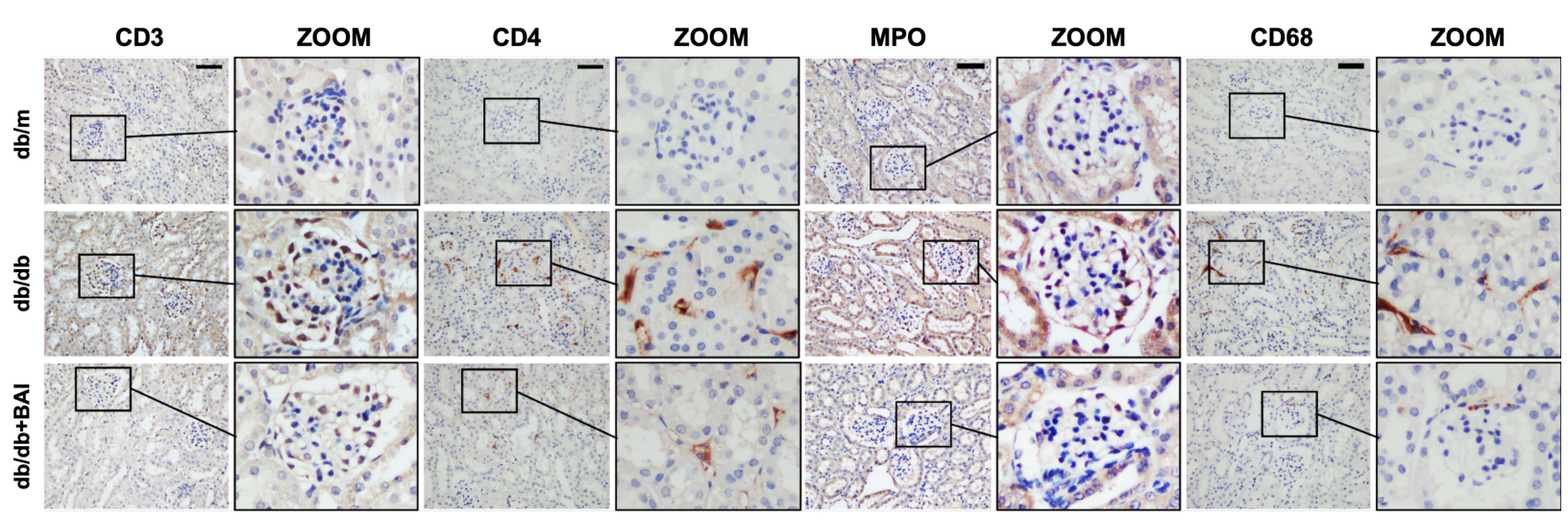

B
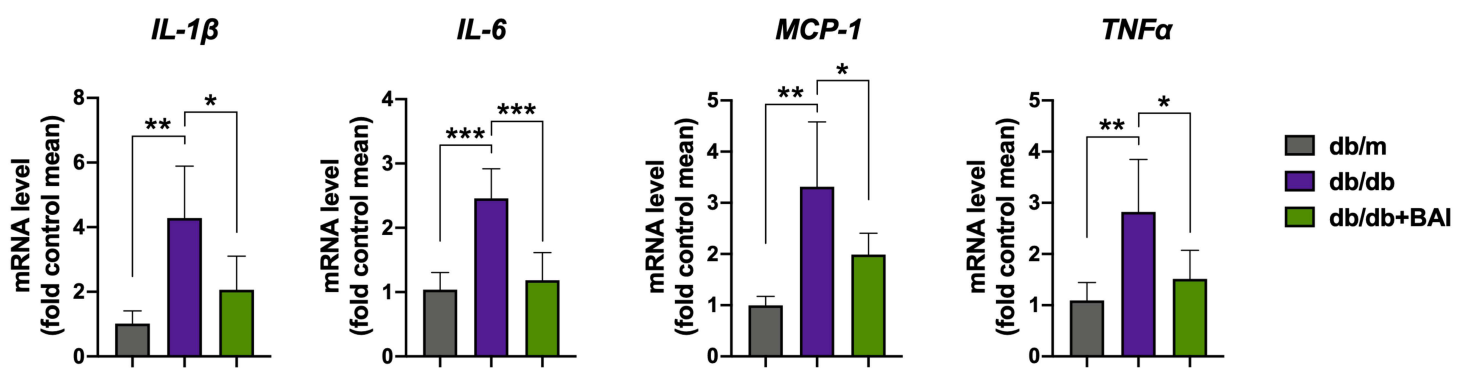

Figure 5 Baicalin administration alleviates inflammatory response in DN. (A) Representative immunohistochemistry staining for CD3 (T-lymphocytes), CD4 (T helper cells), MPO (neutrophils) and CD68 (macrophages). Scale bar, $50 \mu \mathrm{m}$. $(\mathrm{n}=6)(\mathbf{B})$ The mRNA levels of $I L-I \beta$, IL-6, MCP-I and TNF $\alpha$ of different groups. (n=6) All data are presented as means \pm SD. $* p<0.05, * * p<0.01, * * * p<0.001$.

role in the antioxidant system. ${ }^{30}$ In response to oxidative stress, activated Nrf2 translocates into nucleus, and thus leads to the expression of antioxidant response element (ARE)-driven genes, such as heme oxygenase-1 (HO-1) and NAD(P)H: quinone oxidoreductase-1 (NQO-1). ${ }^{31}$ Our results showed that the activation of $\mathrm{Nrf} 2$ signaling and the levels of antioxidant enzymes (HO-1, NQO-1) were obviously inhibited in DN mice, while BAI treatment significantly activated $\mathrm{Nrf} 2$ signaling and increased the protein expression of HO-1 and NQO-1 (Nrf2: Control group vs model group $p<0.001$, BAI group vs model group $p<0.001$; HO-1: Control group vs model group $p=$ 0.0013 , BAI group vs model group $p=0.0011$; NQO-1: Control group vs model group $p<0.001$, BAI group vs model group $p<0.001$ ) (Figure 6A and B). These results indicated that the antioxidant effects of BAI may be associated with the activation of $\mathrm{Nrf} 2$ signaling pathway.

\section{Baicalin Administration Inhibits Activated MAPK Signaling Pathway in DN}

As mentioned above, baicalin administration can exert an ameliorative effect on renal inflammatory response, but the underlying mechanisms remain unclear. To clarify the underlying mechanisms of BAI's anti-inflammatory effects, we applied transcriptomics analysis. In this study, a total of 780 differentially expressed genes (DEGs) were identified in BAI group versus model group, 453 of which were upregulated and 327 were downregulated (Figure 7A). Then, the results of Kyoto Encyclopedia of Genes and Genomes (KEGG) enrichment analysis on DEGs showed that the regulation of MAPK signaling pathway may be the major pathway under BAI administration (Figure 7B).

Since Mitogen-activated protein kinase (MAPK) signaling is well-recognized canonical pro-inflammatory signaling pathway, thus we further examined the activation of MAPK family proteins, including Erk1/2, JNK and P38 signaling. Compared with control group, the phosphorylation of Erk1/2, JNK and P38 was remarkedly increased in model group, suggesting that the MAPK signaling in DN was obviously activated. BAI administration significantly inhibited the increased phosphorylation of Erk1/2, JNK and P38 in DN mice, which proved that baicalin can inhibit activated MAPK signaling pathway in DN (p-JNK: Control group vs model group $p<0.001$, BAI group vs model group $p<$ 0.001; p-P38: Control group vs model group $p<$ 0.001 , BAI group vs model group $p<0.001$; p-ERK: 
A

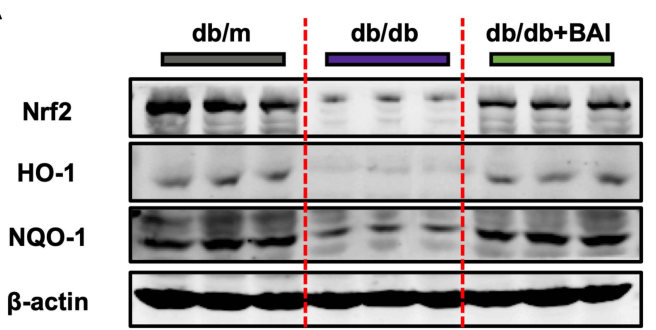

B

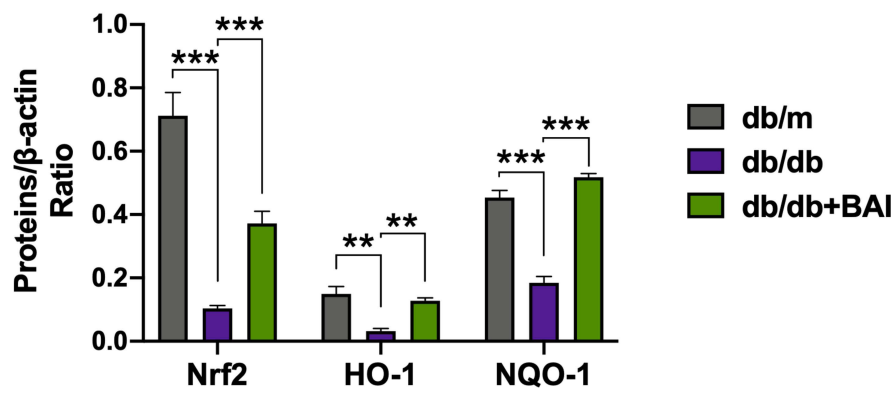

Figure 6 Baicalin administration activates Nrf2-mediated antioxidant pathway in DN. (A) Representative Western blots for Nrf2, HO-I and NQO-I protein expressions in kidney tissue extracts. $(\mathrm{n}=6)(\mathrm{B})$ The quantification of Nrf2, HO-I and NQO-I protein Western blots. All data are presented as means \pm SD. $* * p<0.0 \mathrm{I}, * * * p<0.00 \mathrm{I}$.

A

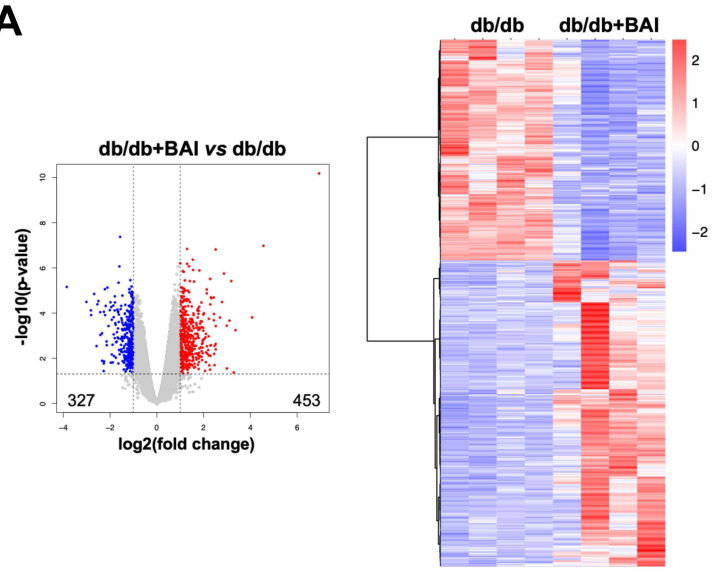

B
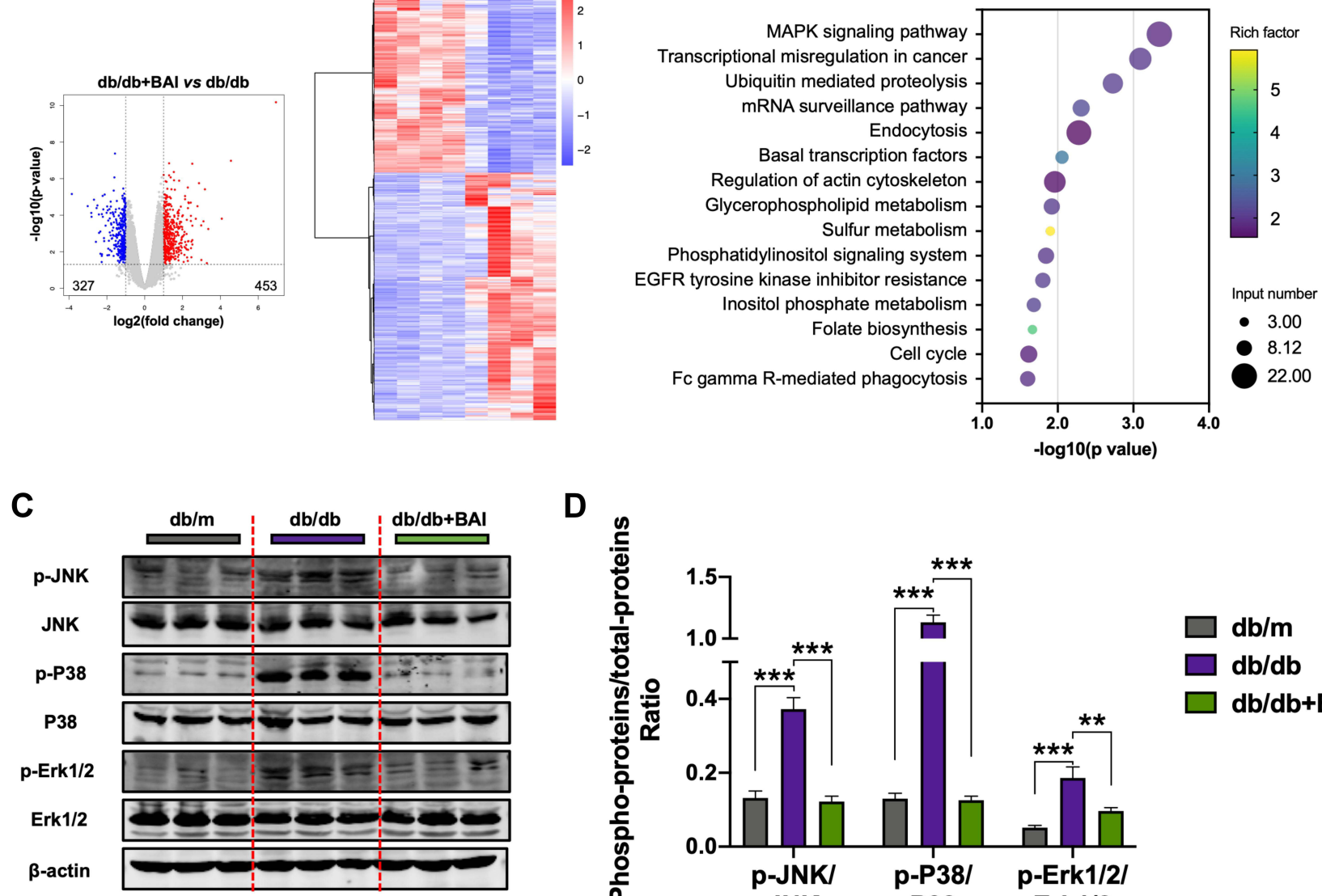

D

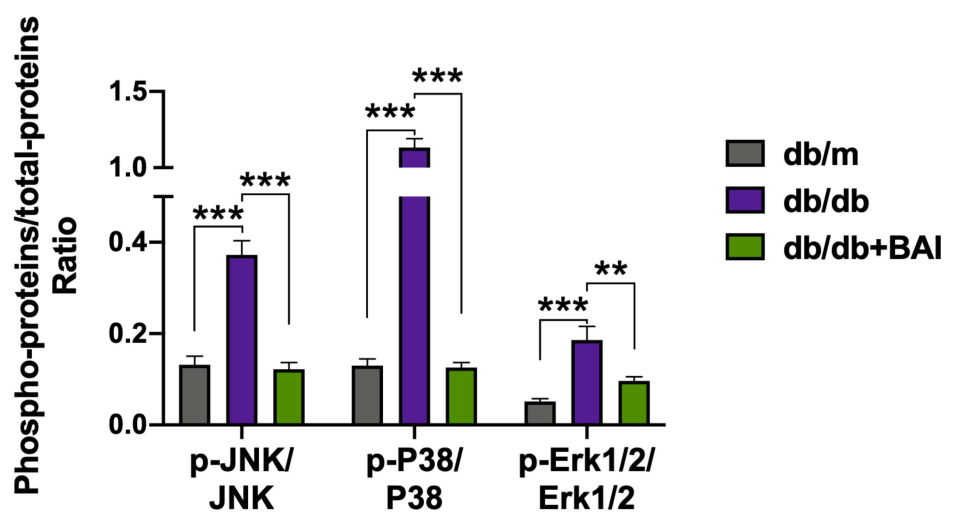

Figure 7 Baicalin administration inhibits activated MAPK signaling pathway in DN. (A) Volcano map showed the number of DEGs in BAI group versus model group; heatmap of these DEGs was also presented. Blue means downregulated gene, red means upregulated gene. ( $n=4)(B)$ KEGG enrichment analysis for DEGs identified by transcriptomics analysis. (C) Representative Western blots for $\mathrm{p}-\mathrm{JNK}$, JNK, p-P38, P38, p-ErkI/2 and Erkl/2 protein expressions in kidney tissue extracts. ( $\mathrm{n}=6$ ) (D) The quantification of $\mathrm{p}-\mathrm{JNK}, \mathrm{JNK}, \mathrm{p}-\mathrm{P} 38, \mathrm{P} 38, \mathrm{p}-\mathrm{ErkI} / 2$ and ErkI/2 protein Western blots. All data are presented as means \pm SD. $* * p<0.0 \mathrm{I}, * * * p<0.00 \mathrm{I}$.

Control group vs model group $p<0.001$, BAI group vs model group $p=0.0091$ ) (Figure 7C and D). Taken together, these results demonstrated that the antiinflammatory effects of BAI may be associated with the inhibition of MAPK signaling pathway.

\section{Discussion}

Although the therapeutic approaches of DN have been explored for decades and proved to be effective to a certain extent, but the population of DN is still growing rapidly. Currently, the main principle for DN treatment is 
the combination of glycemic control and blood pressure control. ${ }^{32}$ In clinic, the application of ACEIs and ARBs in $\mathrm{DN}$ exerts a certain effect in reducing proteinuria, but it is difficult to correct the progressive kidney damage due to its complicated pathogenesis. ${ }^{33}$ So, a large unmet need remains for the development of more effective therapy for DN. Baicalin is derived from traditional Chinese medicinal plant Scutellaria baicalensis Georgi which is also widely used for the prevention and treatment of diabetes and its complications and has been proven effective. ${ }^{34}$ In this present study, we explored the potential effects and underlying mechanisms of BAI on DN. Our results demonstrated that BAI can alleviate oxidative stress, by activating Nrf2-mediated antioxidant pathway, and inflammation, by inhibiting MAPK signaling pathway, thereby protecting against DN.

Previous studies have indicated that BAI can prevent DN via suppressing renal fibrosis. ${ }^{21-23}$ As we all know, renal fibrosis is not an initial factor in the development of CKD but may play an important role in the later stage. On the contrary, renal fibrosis is usually induced under chronic stress environment. ${ }^{35}$ However, BAI's investigations on the upstream events of $\mathrm{DN}$ : oxidative stress and inflammation are still lacking. Therefore, we conducted this study. Usually, spontaneous DM mice (db/db or ob/ ob) will develop typical DN symptom and pathological changes including fibrosis at 20 weeks or older. So, in our work, we applied relatively short intervention time (12 weeks) to avoid the influence of fibrosis. As expected, our DN model did not show significant tubulointerstitial fibrosis (Supplementary Figure 4), but showed typical microalbuminuria and histopathological changes of DN. This early DN phenotype is more suitable for our purpose. In line with that, there was also no kidney dysfunction presented in our DN mice.

Oxidative stress is considered to be a key pathway in the diabetic kidney damage under metabolic and hemodynamic abnormalities. ${ }^{36}$ ROS family includes molecular oxygen and its derivatives, like superoxide anion $\left(\mathrm{O}^{2-}\right)$, hydroxyl radical $(\mathrm{HO} \bullet)$, hydrogen peroxide $\left(\mathrm{H}_{2} \mathrm{O}_{2}\right)$, peroxynitrite $\left(\mathrm{ONOO}^{-}\right)$, hypochlorous acid $(\mathrm{HClO})$, nitric oxide (NO) and lipid radicals. Studies found that the main sources of ROS include mitochondrial respiratory chain, xanthine oxidase, NADH/NADPH oxidases, NO synthase and some other hemoproteins. Oxidative stress develops when excessive ROS exceeds endogenous antioxidant system, and then bioactive molecules such as carbohydrates, proteins, lipids and DNA will be oxidized, contributing to cell damage. ${ }^{37}$ Due to the diverse sources of ROS, it is conceivable that hyperglycemia-mediated ROS generation involves various different mechanisms, ${ }^{38}$ in which diacylglycerol (DAG)-protein kinase $\mathrm{C}$ (PKC)and NADPH-oxidase axis is suggested as the major dangerous metabolic route. ${ }^{39}$ Whatever, one thing is certain, that is, hyperglycemia will lead to oxidative stress injury. This point was confirmed in our study. DN mice showed a significant oxidative stress state with decreased antioxidant enzymes (GSH-PX, SOD and CAT) and increased MDA. BAI treatment successfully prevented excessive ROS production in kidney and reduced tissue damage. From these results, we can confirm that BAI alleviates hyperglycemia-induced oxidative stress in DN. In endogenous antioxidant defense system, transcription factor Nrf2 is believed to be the most important signaling molecular. Under oxidative stress stimulation, activated Nrf2 protein translocates into nucleus, and binds to the ARE sequence of downstream gene promoter, finally induces the gene expressions. ${ }^{40}$ The transcriptional regulation of HO-1, NQO-1, glutathione S-transferase (GST) and glutathione peroxidase (GPx-1) are all controlled by $\mathrm{Nrf} 2$ activation. $^{41}$ Therefore, activating Nrf2 signaling has been regarded as an effective approach for the treatment of diabetic complications. Many studies have confirmed that the activation of Nrf2 signaling improves DN though relieving oxidative stress. ${ }^{42,43}$ Therefore, we assumed that BAI exerts the antioxidant effects by activating Nrf2mediated antioxidant pathway. Consistently, our data found that BAI treatment indeed significantly activated Nrf2 signaling and increased its downstream protein expression (HO-1 and NQO-1). Together, these results demonstrated that BAI alleviates oxidative stress in DN by activating Nrf2-mediated antioxidant pathway.

In addition to oxidative stress, inflammation undoubtedly constitutes another crucial promoter in the pathogenesis of DN., Lots of studies have indicated that the activation of inflammation signaling and inflammatory cell infiltration is essential to the development of DN. ${ }^{44}$ Hence, we investigated the effects of BAI on the inflammatory responses, and found that BAI significantly inhibits the infiltration of various inflammatory cells (T-lymphocytes, $\mathrm{T}$ helper cells, neutrophils and macrophages) and the expression of pro-inflammatory cytokines like IL-1 $\beta$, IL-6, MCP-1 and TNF $\alpha$. Then, renal mRNAsequencing was applied to clarify the potential mechanisms of BAI's anti-inflammatory effects on DN. We found that BAI's anti-inflammatory effects may attribute to its 
inhibitory activity on the classic pro-inflammatory signaling MAPK pathway. As a canonical pro-inflammatory pathway, excessive activation of MAPK pathway has been proven to be strongly associated with many inflammatory diseases. In fact, many MAPK inhibitors have been evaluated preclinically in a wide range of disease models with a considerable efficacy. ${ }^{45}$ Our results were consistent with many previous works. Under chronic hyperglycemia, MAPK pathway can be activated, thus leading to local inflammatory response. ${ }^{46}$ A number of studies reported that many natural products, including celastrol, ${ }^{47}$ ginsenoside, ${ }^{48}$ and apigenin, ${ }^{49}$ could suppress renal inflammation in DN by inhibiting MAPK signaling pathway. As a medicinal plant, Scutellaria baicalensis Georgi (S. baicalensis) has been applied in China for centuries. To date, there is no report of severe side effects from in vivo studies of BAI. On the contrary, BAI as a natural flavonoid is widely used in cosmetics. Therefore, combined with our results, it was justified to conclude that BAI can act as a natural antioxidant and anti-inflammation agent in clinical practice with high safety spectrum.

As mentioned before, there is a complex interaction between oxidative stress and inflammation. This relationship is also existing between Nrf2 and MAPK signaling pathways. Studies have indicated that the activation of Erk1/2 can inhibit Nrf2 activity, thereby contributing to oxidative stress. ${ }^{43}$ Meanwhile, the inhibition of MAPK signaling can enhance Nrf2 activity and HO-1 production. ${ }^{50}$ The MAPK family can affect Nrf2 activity, and in turn, Nrf2 activity can also regulate the activation of MAPK signaling pathway. It was found that the forced activation (adenoviral overexpression) of $\mathrm{Nrf} 2$ can significantly suppress Erk1/2 activation. ${ }^{43}$ On the contrary, the function loss of Nrf2 led to the activation of MAPK signaling and increased inflammation. ${ }^{51}$ In a word, abnormal Nrf2 and MAPK signaling transductions together promote the development of DN by the modulation of oxidative stress and inflammation. Although the complex crosstalk between these mechanisms needs to be further clarified, the promotive direction of these factors in DN is definite. Whatever, our findings demonstrated that BAI not only activates Nrf2 signaling pathway but also inhibits MAPK signaling pathway, and then in concert alleviates upstream oxidative stress and inflammation, thereby preventing DN (Figure 8).

This work verified that both oxidative stress and inflammation are closely related to the pathogenesis of $\mathrm{DN}$, and our results introduced BAI as an effective agent for the treatment of DN. Moreover, due to many diabetic complications share common upstream pathogenesis, published evidences have demonstrated that BAI can also improve diabetic cardiac diseases and diabetic retinopathy via inhibiting oxidative stress and MAPK-inflammation pathway. ${ }^{52,53}$ We believe that this feature can also be deduced from other natural products with both antioxidant and anti-inflammatory properties during the treatment of diabetic complications. Additionally, these findings provided compelling evidence that Nrf2 and MAPK signaling pathways were promising targets for diabetic nephropathy.

\section{Limitations of the Present Study}

There are still some limitations of our work. This current study indicated that BAI could treat $\mathrm{DN}$ by alleviating oxidative stress and inflammation, but based on our in vivo evidence, we cannot point to a specific cell type. So, in this stage, we did not perform in vitro study. The pathogenesis of DN is complex and associated with many specific cell types, like podocyte, glomerular endothelial cell, mesangial cell and tubule epithelial cell. Overall, many studies have revealed that oxidative stress and inflammation can affect all these cells and together contribute to the progression of DN. Therefore, we hope more comprehensive targeted-investigations will be conducted in the future. In addition, for RNA-seq, we applied two group-based (drug vs model group) analysis, not three group-based (combine model vs control group with drug vs model group) analysis. Although two group- and three group-based analyses are both widely used in pharmacological transcriptomics analysis, ${ }^{54-57}$ the latter is more precise. For two group-based analysis, we can tell the overall effects of drug on certain disease model by comparing drug group to model group; For three group-based analysis, we can tell the corrective effects of drug on certain disease model by integrating model vs control group with drug vs model group. We humbly believed that after confirming the therapeutic effects of drug (BAI) on certain disease model (DN), the two groupbased analysis is closely to the three group-based analysis. Therefore, at that time, we only conducted RNA-seq for drug and model groups in this study. Although the RNAseq result is logical and convincing, more precise solutions are more in line with scientific requirements.

\section{Conclusion}

In conclusion, this study explored the potential effects and underlying mechanisms of BAI on DN. These results 


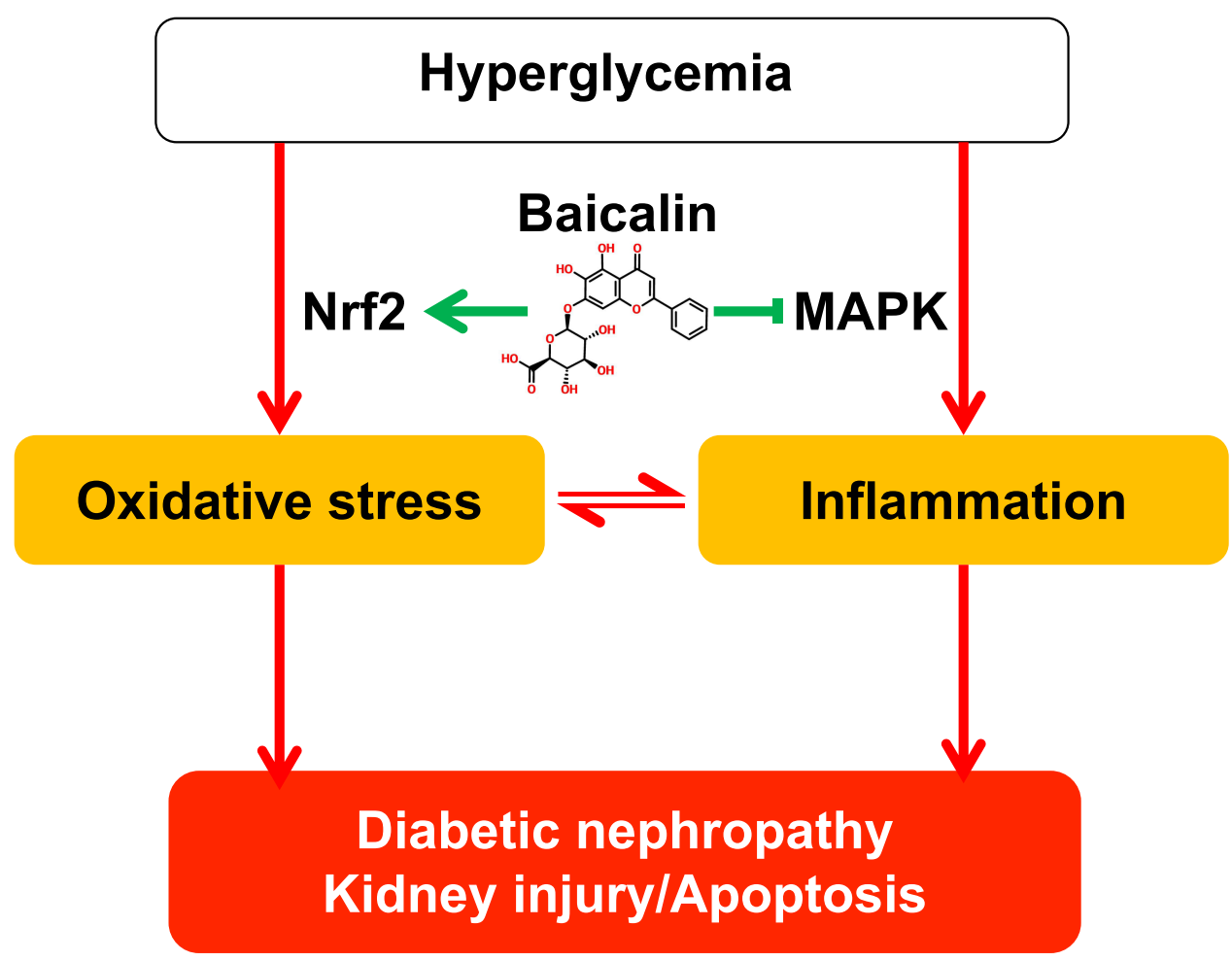

Figure 8 Baicalin alleviates oxidative stress and inflammation in diabetic nephropathy via Nrf2 and MAPK signaling pathway. In DM, persistent hyperglycemia can induce oxidative stress and inflammation, which play essential roles in the development and progression of diabetic nephropathy. BAI administration can treat DN by alleviating oxidative stress and inflammation, and its underlying mechanisms were associated with the activation of Nrf2-mediated antioxidant signaling pathway and the inhibition of MAPK-mediated inflammatory signaling pathway.

indicated that BAI also has a significant impact on the upstream events in the pathogenesis of $\mathrm{DN}$, rather than concentrating on later fibrogenesis. Our data demonstrated that BAI can treat DN by alleviating oxidative stress and inflammation, and its underlying mechanisms were associated with the activation of Nrf2-mediated antioxidant signaling pathway and the inhibition of MAPK-mediated inflammatory signaling pathway.

\section{Abbreviations}

DN, Diabetic nephropathy; BAI, Baicalin; CKD, chronic kidney disease; DM, diabetes mellitus; ESRD, End-stage renal disease; ACEIs, Angiotensin-converting enzyme inhibitors; ARBs, Angiotensin receptor blockades; ROS, Reactive oxygen species; FBG, Fasting blood glucose; GTT, Glucose tolerance test; ITT, Insulin tolerance test; HOMA-IR, Homeostasis model assessment insulin resistance; BUN, Urea nitrogen; $\mathrm{Cr}$, Creatinine; AER, Urine albumin excretion rate; GSH-PX, Glutathione peroxidase; SOD, Superoxide dismutase; CAT, Catalase; MDA, Malonaldehyde; GO, Gene ontology; KEGG, Kyoto Encyclopedia of Genes and Genomes; DEG, Differentially expressed gene; SEM, Scanning electron microscopy; TEM,
Transmission electron microscopy; GBM, Glomerular basement membrane; TUNEL, Terminal deoxynucleotidyl transferase dUTP nick end labeling; ARE, Antioxidant response element; Nrf2, Nuclear factor erythroid-derived 2-related factor 2; HO-1, heme oxygenase-1; NQO-1, NAD $(\mathrm{P}) \mathrm{H}$ : quinone oxidoreductase-1; MAPK, Mitogen-activated protein kinase; $\mathrm{O}^{2-}$, Superoxide anion; $\mathrm{HO} \cdot$, Hydroxyl radical; $\mathrm{H}_{2} \mathrm{O}_{2}$, Hydrogen peroxide; $\mathrm{ONOO}^{-}$, Peroxynitrite; $\mathrm{HClO}$, Hypochlorous acid; NO, Nitric oxide; DAG, Diacylglycerol; PKC, Protein kinase C; GST, Glutathione S-transferase; GPx-1, Glutathione peroxidase.

\section{Data Sharing Statement}

The datasets used and/or analysed during the current study are available from the corresponding author on reasonable request.

\section{Ethics Approval and Informed Consent}

All of the procedures were reviewed and approved by the Animal Ethics Committee of Tongji Hospital of Tongji Medical College of Huazhong University of Science and 
Technology according to the Guideline for Ethical Review of Animal Welfare (GB/T 35892-2018).

\section{Author Contributions}

All authors contributed to data analysis, drafting or revising the article, gave final approval of the version to be published, agreed to the submitted journal, and agreed to be accountable for all aspects of the work.

\section{Funding}

This work was supported by the National Natural Science Foundation of China (No. 81974567), and TCM Modernization Research of National Key Research and Development Program (No. 2018YFC1704202).

\section{Disclosure}

The authors declare no conflict of interest.

\section{References}

1. Zhang L, Long J, Jiang $\mathrm{W}$, et al. Trends in chronic kidney disease in China. $N$ Engl J Med. 2016;375(9):905-906. doi:10.1056/ NEJMc1602469

2. Palmer SC, Mavridis D, Navarese E, et al. Comparative efficacy and safety of blood pressure-lowering agents in adults with diabetes and kidney disease: a network meta-analysis. Lancet. 2015;385 (9982):2047-2056. doi:10.1016/S0140-6736(14)62459-4

3. Ni Z, Guo L, Liu F, Olatunji OJ, Yin M. Allium tuberosum alleviates diabetic nephropathy by supressing hyperglycemia-induced oxidative stress and inflammation in high fat diet/streptozotocin treated rats. Biomed Pharmacother. 2019;112:108678. doi:10.1016/j. biopha.2019.108678

4. Zhong Y, Lee K, Deng Y, et al. Arctigenin attenuates diabetic kidney disease through the activation of PP2A in podocytes. Nat Commun. 2019;10(1):4523. doi:10.1038/s41467-019-12433-w

5. Wang W, Sun W, Cheng Y, Xu Z, Cai L. Role of sirtuin-1 in diabetic nephropathy. J Mol Med. 2019;97(3):291-309. doi:10.1007/s00109019-01743-7

6. Wada J, Makino H. Innate immunity in diabetes and diabetic nephropathy. Nat Rev Nephrol. 2016;12(1):13-26. doi:10.1038/ nrneph.2015.175

7. Gnudi L, Coward RJM, Long DA. Diabetic nephropathy: perspective on novel molecular mechanisms. Trends Endocrinol Metab. 2016;27 (11):820-830. doi:10.1016/j.tem.2016.07.002

8. Jha JC, Banal C, Chow BS, Cooper ME, Jandeleit-Dahm K. Diabetes and kidney disease: role of oxidative stress. Antioxid Redox Signal. 2016;25(12):657-684. doi:10.1089/ars.2016.6664

9. Sagoo MK, Gnudi L. Diabetic nephropathy: is there a role for oxidative stress? Free Radic Biol Med. 2018;116:50-63. doi:10.1016/j.freeradbiomed.2017.12.040

10. Sies H. Oxidative stress: a concept in redox biology and medicine. Redox Biol. 2015;4:180-183. doi:10.1016/j.redox.2015.01.002

11. Reuter S, Gupta SC, Chaturvedi MM, Aggarwal BB. Oxidative stress, inflammation, and cancer: how are they linked? Free Radic Biol Med. 2010;49(11):1603-1616. doi:10.1016/j.freeradbiomed.20 10.09 .006
12. Wu F, Yang $\mathrm{X}, \mathrm{Hu} \mathrm{M}$, et al. Wu-Mei-Wan prevents high-fat diet-induced obesity by reducing white adipose tissue and enhancing brown adipose tissue function. Phytomedicine. 2020;76:153258. doi:10.1016/j.phymed.2020.153258

13. Dandekar A, Mendez R, Zhang K. Cross talk between ER stress, oxidative stress, and inflammation in health and disease. Methods Mol Biol. 2015;1292:205-214. doi:10.1007/978-1-4939-2522-3_15

14. Luc K, Schramm-Luc A, Guzik TJ, Mikolajczyk TP. Oxidative stress and inflammatory markers in prediabetes and diabetes. $J$ Physiol Pharmacol. 2019;70(6). doi:10.26402/jpp.2019.6.01

15. Yaribeygi H, Sathyapalan T, Atkin SL, Sahebkar A. Molecular mechanisms linking oxidative stress and diabetes mellitus. Oxid Med Cell Longev. 2020;2020:8609213. doi:10.1155/2020/8609213

16. Wang E, Wang L, Ding R, et al. Astragaloside IV acts through multi-scale mechanisms to effectively reduce diabetic nephropathy. Pharmacol Res. 2020;157:104831. doi:10.1016/j.phrs.2020.104831

17. Bao L, Li J, Zha D, et al. Chlorogenic acid prevents diabetic nephropathy by inhibiting oxidative stress and inflammation through modulation of the Nrf2/HO-1 and NF-kB pathways. Int Immunopharmacol. 2018;54:245-253. doi:10.1016/j.intimp.2017.11.021

18. Lee D, Ko WK, Hwang DS, et al. Use of baicalin-conjugated gold nanoparticles for apoptotic induction of breast cancer cells. Nanoscale Res Lett. 2016;11(1):381. doi:10.1186/s11671-016-1586-3

19. Li HT, Wu XD, Davey AK, Wang J. Antihyperglycemic effects of baicalin on streptozotocin - nicotinamide induced diabetic rats. Phytother Res. 2011;25(2):189-194. doi:10.1002/ptr.3238

20. He P, Wu Y, Shun J, Liang Y, Cheng M, Wang Y. Baicalin ameliorates liver injury induced by chronic plus binge ethanol feeding by modulating oxidative stress and inflammation via CYP2E1 and NRF2 in mice. Oxid Med Cell Longev. 2017;2017:4820414. doi:10.1155/ 2017/4820414

21. Zhang S, Xu L, Liang R, Yang C, Wang P. Baicalin suppresses renal fibrosis through microRNA-124/TLR4/NF-kappaB axis in streptozotocin-induced diabetic nephropathy mice and high glucose-treated human proximal tubule epithelial cells. J Physiol Biochem. 2020;76(3):407-416. doi:10.1007/s13105-020-00747-z

22. Zheng XP, Nie Q, Feng J, et al. Kidney-targeted baicalin-lysozyme conjugate ameliorates renal fibrosis in rats with diabetic nephropathy induced by streptozotocin. BMC Nephrol. 2020;21(1):174. doi:10.1186/s12882-020-01833-6

23. Nam JE, Jo SY, Ahn CW, Kim YS. Baicalin attenuates fibrogenic process in human renal proximal tubular cells (HK-2) exposed to diabetic milieu. Life Sci. 2020;254:117742. doi:10.1016/j.1fs.2020.117742

24. Fan Y, Yi Z, D'Agati VD, et al. Comparison of kidney transcriptomic profiles of early and advanced diabetic nephropathy reveals potential new mechanisms for disease progression. Diabetes. 2019;68 (12):2301-2314. doi:10.2337/db19-0204

25. Yang M, Kan L, Wu L, Zhu Y, Wang Q. Effect of baicalin on renal function in patients with diabetic nephropathy and its therapeutic mechanism. Exp Ther Med. 2019;17(3):2071-2076. doi:10.3892/ etm.2019.7181

26. Cai Y, Li S, Li T, Zhou R, Wai AT, Yan R. Oral pharmacokinetics of baicalin, wogonoside, oroxylin A 7-O-beta-d-glucuronide and their aglycones from an aqueous extract of scutellariae radix in the rat. $J$ Chromatogr B Analyt Technol Biomed Life Sci. 2016;1026:124-133. doi:10.1016/j.jchromb.2015.11.049

27. Dai J, Liang K, Zhao S, et al. Chemoproteomics reveals baicalin activates hepatic CPT1 to ameliorate diet-induced obesity and hepatic steatosis. Proc Natl Acad Sci U S A. 2018;115(26):E5896-E5905. doi:10.1073/pnas.1801745115

28. Sifuentes-Franco S, Padilla-Tejeda DE, Carrillo-Ibarra S, MirandaDiaz AG. Oxidative stress, apoptosis, and mitochondrial function in diabetic nephropathy. Int $J$ Endocrinol. 2018;2018:1875870. doi:10.1155/2018/1875870 
29. Droge W. Free radicals in the physiological control of cell function. Physiol Rev. 2002;82(1):47-95. doi:10.1152/physrev.00018.2001

30. Tonelli C, Chio IIC, Tuveson DA. Transcriptional regulation by Nrf2. Antioxid Redox Signal. 2018;29(17):1727-1745. doi:10.1089/ ars. 2017.7342

31. Loboda A, Damulewicz M, Pyza E, Jozkowicz A, Dulak J. Role of $\mathrm{Nrf}$ /HO-1 system in development, oxidative stress response and diseases: an evolutionarily conserved mechanism. Cell Mol Life Sci. 2016;73(17):3221-3247. doi:10.1007/s00018-016-2223-0

32. Umanath K, Lewis JB. Update on diabetic nephropathy: core curriculum 2018. Am J Kidney Dis. 2018;71(6):884-895. doi:10.1053/j. ajkd.2017.10.026

33. Zhang XT, Wang G, Ye LF, et al. Baicalin reversal of DNA hypermethylation-associated klotho suppression ameliorates renal injury in type 1 diabetic mouse model. Cell Cycle. 2020;19 (23):3329-3347. doi:10.1080/15384101.2020.1843815

34. Shin NR, Gu N, Choi HS, Kim H. Combined effects of Scutellaria baicalensis with metformin on glucose tolerance of patients with type 2 diabetes via gut microbiota modulation. Am J Physiol Endocrinol Metab. 2020;318(1):E52-E61. doi:10.1152/ajpendo.00221.2019

35. Wu F, Shao Q, Hu M, et al. Wu-Mei-Wan ameliorates chronic colitis-associated intestinal fibrosis through inhibiting fibroblast activation. $J$ Ethnopharmacol. 2020;252:112580. doi:10.1016/j. jep.2020.112580

36. Badal SS, Danesh FR. New insights into molecular mechanisms of diabetic kidney disease. Am J Kidney Dis. 2014;63(2 Suppl 2):S63S83. doi:10.1053/j.ajkd.2013.10.047

37. Forbes JM, Coughlan MT, Cooper ME. Oxidative stress as a major culprit in kidney disease in diabetes. Diabetes. 2008;57 (6):1446-1454. doi:10.2337/db08-0057

38. Duchen MR. Roles of mitochondria in health and disease. Diabetes. 2004;53(Suppl 1):S96-S102. doi:10.2337/diabetes.53.2007.s96

39. Nogueira-Machado JA, Chaves MM. From hyperglycemia to AGE-RAGE interaction on the cell surface: a dangerous metabolic route for diabetic patients. Expert Opin Ther Targets. 2008;12 (7):871-882. doi:10.1517/14728222.12.7.871

40. Singh S, Vrishni S, Singh BK, Rahman I, Kakkar P. Nrf2-ARE stress response mechanism: a control point in oxidative stress-mediated dysfunctions and chronic inflammatory diseases. Free Radic Res. 2010;44(11):1267-1288. doi:10.3109/10715762.2010.507670

41. Nguyen T, Nioi P, Pickett CB. The Nrf2-antioxidant response element signaling pathway and its activation by oxidative stress. J Biol Chem. 2009;284(20):13291-13295. doi:10.1074/jbc.R900010200

42. Adelusi TI, Du L, Hao M, et al. Keap1/Nrf2/ARE signaling unfolds therapeutic targets for redox imbalanced-mediated diseases and diabetic nephropathy. Biomed Pharmacother. 2020;123:109732. doi:10.1016/j.biopha.2019.109732

43. Tan Y, Ichikawa T, Li J, et al. Diabetic downregulation of Nrf2 activity via ERK contributes to oxidative stress-induced insulin resistance in cardiac cells in vitro and in vivo. Diabetes. 2011;60 (2):625-633. doi: $10.2337 / \mathrm{db} 10-1164$

44. Galkina E, Ley K. Leukocyte recruitment and vascular injury in diabetic nephropathy. $J$ Am Soc Nephrol. 2006;17(2):368-377. doi:10.1681/ASN.2005080859
45. Yong HY, Koh MS, Moon A. The p38 MAPK inhibitors for the treatment of inflammatory diseases and cancer. Expert Opin Investig Drugs. 2009;18(12):1893-1905. doi:10.1517/13543780903321490

46. Sakai N, Wada T, Furuichi K, et al. Involvement of extracellular signal-regulated kinase and p38 in human diabetic nephropathy. Am J Kidney Dis. 2005;45(1):54-65. doi:10.1053/j.ajkd.2004.08.039

47. Zhang M, Chen Y, Yang MJ, et al. Celastrol attenuates renal injury in diabetic rats via MAPK/NF-kappaB pathway. Phytother Res. 2019;33 (4):1191-1198. doi:10.1002/ptr.6314

48. Zhu Y, Zhu C, Yang H, Deng J, Fan D. Protective effect of ginsenoside Rg5 against kidney injury via inhibition of NLRP3 inflammasome activation and the MAPK signaling pathway in high-fat diet/ streptozotocin-induced diabetic mice. Pharmacol Res. 2020;155:104746. doi:10.1016/j.phrs.2020.104746

49. Malik S, Suchal K, Khan SI, et al. Apigenin ameliorates streptozotocin-induced diabetic nephropathy in rats via MAPK-NFkappaB-TNF-alpha and TGF-beta1-MAPK-fibronectin pathways. $\mathrm{Am}$ J Physiol Renal Physiol. 2017;313(2):F414-F422. doi:10.1152/ ajprenal.00393.2016

50. Ko WC, Shieh JM, Wu WB. P38 MAPK and Nrf2 activation mediated naked gold nanoparticle induced heme oxygenase-1 expression in rat aortic vascular smooth muscle cells. Arch Med Res. 2020;51(5):388-396. doi:10.1016/j.arcmed.2020.04.015

51. Jiang T, Tian F, Zheng H, et al. Nrf2 suppresses lupus nephritis through inhibition of oxidative injury and the NF-kappaB-mediated inflammatory response. Kidney Int. 2014;85(2):333-343. doi:10.1038/ki.2013.343

52. Sheng X, Wang J, Guo J, et al. Effects of baicalin on diabetic cardiac autonomic neuropathy mediated by the $\mathrm{P} 2 \mathrm{Y} 12$ receptor in rat stellate ganglia. Cell Physiol Biochem. 2018;46(3):986-998. doi:10.1159/ 000488828

53. Dai C, Jiang S, Chu C, Xin M, Song X, Zhao B. Baicalin protects human retinal pigment epithelial cell lines against high glucose-induced cell injury by up-regulation of microRNA-145. Exp Mol Pathol. 2019;106:123-130. doi:10.1016/j.yexmp.2019.01.002

54. Qiao YL, Zhou JJ, Liang JH, et al. Uncaria rhynchophylla ameliorates unpredictable chronic mild stress-induced depression in mice via activating 5-HT1A receptor: insights from transcriptomics. Phytomedicine. 2021;81:153436. doi:10.1016/j.phymed.2020.153436

55. Zhang C, Qiao S, Wu J, et al. A new insulin-sensitive enhancer from Silene viscidula, WPTS, treats type 2 diabetes by ameliorating insulin resistance, reducing dyslipidemia, and promoting proliferation of islet beta cells. Pharmacol Res. 2021;165:105416. doi:10.1016/j. phrs.2020.105416

56. Ding Z, Zhong R, Yang Y, et al. Systems pharmacology reveals the mechanism of activity of Ge-Gen-Qin-Lian decoction against LPS-induced acute lung injury: a novel strategy for exploring active components and effective mechanism of TCM formulae. Pharmacol Res. 2020;156:104759. doi:10.1016/j.phrs.2020.104759

57. Mohammadtursun N, Li Q, Abuduwaki M, et al. Loki zupa alleviates inflammatory and fibrotic responses in cigarette smoke induced rat model of chronic obstructive pulmonary disease. Chin Med. 2020;15:92. doi:10.1186/s13020-020-00373-3
Drug Design, Development and Therapy

\section{Publish your work in this journal}

Drug Design, Development and Therapy is an international, peerreviewed open-access journal that spans the spectrum of drug design and development through to clinical applications. Clinical outcomes, patient safety, and programs for the development and effective, safe, and sustained use of medicines are a feature of the journal, which has also been accepted for indexing on PubMed Central. The manuscript management system is completely online and includes a very quick and fair peer-review system, which is all easy to use. Visit http://www. dovepress.com/testimonials.php to read real quotes from published authors. 